\title{
Maternal and paternal depressive symptoms and children's emotional problems at the age of 2 and 5 years: a longitudinal study
}

\author{
Johanna T. Pietikäinen, ${ }^{1,2}$ (D) Olli Kiviruusu, ${ }^{1}$ Anneli Kylliäinen, ${ }^{3}$ Pirjo Pölkki, ${ }^{4}$ \\ Outi Saarenpää-Heikkilä, ${ }^{5,6}$ Tiina Paunio,, ${ }^{1,2}$ and E. Juulia Paavonen ${ }^{1,7}$ \\ ${ }^{1}$ Department of Public Health Solutions, National Institute for Health and Welfare, Helsinki, Finland; ${ }^{2}$ Psychiatry, \\ University of Helsinki and Helsinki University Hospital, Helsinki, Finland; ${ }^{3}$ Psychology, Faculty of Social Sciences, \\ University of Tampere, Tampere, Finland; ${ }^{4}$ Department of Social Sciences, University of Eastern Finland, Kuopio, \\ Finland; ${ }^{5}$ Pediatric Clinics, Tampere University Hospital, Tampere, Finland; ${ }^{6}$ Tampere Centre for Child Health \\ Research, University of Tampere and Tampere University Hospital, Tampere, Finland; ${ }^{7}$ Pediatric Research Center, \\ Child Psychiatry, University of Helsinki and Helsinki University Hospital, Helsinki, Finland
}

\begin{abstract}
Background: Maternal and paternal depressive symptoms are related to children's emotional problems, but their combined effect remains unclear. Here, we constructed four parental longitudinal depressive symptom trajectory groups and studied their associations with children's emotional problems at the age of 2 and 5 years. Methods: We did an assessment of maternal and paternal depressive symptoms (gestational week 32, as well as 3, 8 and 24 months postnatally) and children's emotional problems at ages two $(N=939)$ and five $(N=700)$ in the CHILD-SLEEP cohort. Three separate maternal and paternal depressive symptom trajectories based on latent profile analysis were combined to form four parental depressive symptom trajectory groups. We compared groups with a general linear model, with children's emotional (total, internalizing and externalizing) - problem scores serving as the dependent variables. Results: At both ages, combined parental depressive symptom trajectories were associated with children's emotional problems: effect sizes were medium for total and small for other domains. According to post hoc comparisons, children whose mothers or both parents had persistent depressive symptoms had significantly more total, externalizing and internalizing problems than did children who had neither parent nor only the father showing depressive symptoms. A higher (and persistent) level of maternal depressive symptoms was related to a higher level of these children's emotional problems, a pattern not evident with paternal depressive symptoms. In all analyses, the interaction effect was nonsignificant between parental trajectories and child gender. Conclusions: Findings suggest that an absence of depressive symptoms in their fathers cannot compensate for the adverse effects of maternal depressive symptoms upon their children. Moreover, paternal depressive symptoms alone do not lead to increased risk for emotional problems in these 2- and 5-year-old children. In contrast, even subclinical levels of maternal depressive symptoms in late pregnancy are associated with increased risk for their children's experiencing internalizing and externalizing emotional problems. Keywords: Depression; maternal depression; perinatal; longitudinal studies; child development.
\end{abstract}

\section{Introduction}

Some of the greatest risk factors for children's mental health symptoms are parental psychiatric disorders. Among these, postpartum depression (PPD) affects approximately 13\%-19\% of mothers (O'Hara \& McCabe, 2013) and approximately $8 \%$ of fathers during the first year of their child's life (Cameron, Sedov, \& Tomfohr-Madsen, 2016). One estimate is that in the United States, at least 15 million children under age 18 live with an adult who has experienced major depression in the previous year (England \& Sim, 2009). Any intergenerational transmission of depression and other psychiatric disorders is thus important in terms of prevention. One suggestion is that maternal depression during pregnancy may create a 'meta-plastic state' in the brain of the foetus, which may further sensitize the infant to the quality of postnatal care (O'Donnell \&

Conflict of interest statement: No conflicts of interest.
Meaney, 2017). The early postpartum months and the first year is indeed an important and sensitive period when the child is particularly susceptible to harmful effects of maternal depression (Bagner, Pettit, Lewinsohn, \& Seeley, 2010; Murray et al., 1999).

A meta-analysis by Goodman et al. (2011) of the effects of maternal depression on offspring concluded that maternal depression is associated with children's externalizing (overactivity, defiance, aggression) and internalizing symptoms (anxiety, depression) and general psychopathology, but with small effect sizes. Weissman, Wickramaratne et al. $(2006,2016)$ followed the 147 offspring of depressed parents for over 30 years, showing that during a 30year follow-up, children with one or both parents who had been depressed had approximately a threefold risk for developing a major depressive disorder, anxiety disorder or substance-use disorder themselves, when compared to the risk for offspring of nondepressed parents. The adverse effects of 
parental depression tend to appear even in the third generation (Weissman, Berry et al., 2016). A limitation in these studies has been difficulty in accounting for persistence of parental depression; the child's symptoms (and diagnoses), in fact, closely reflect the status of concurrent maternal depression (Weissman, Pilowsky et al., 2006).

Several longitudinal studies have shown that maternal high depressive symptom trajectories are associated with children's emotional problems (Campbell, Matestic, von Stauffenberg, Mohan, \& Kirchner, 2007; Campbell, Morgan-Lopez, Cox, \& McLoyd, 2009; Cents et al., 2013; Giallo, Woolhouse, Gartland, Hiscock, \& Brown, 2015; Kingston et al., 2018; Park, Brain, Grunau, Diamond, \& Oberlander, 2018; Van Der Waerden et al., 2015). These reports typically identify three to six stable groups of depressive mothers. The offspring of the mothers in the higher depressive symptom trajectory groups - and those with more severe persistent depressive symptoms - have significantly more internalizing and externalizing problems than do those with mothers showing lower levels of persistent depression or no depression. Moreover, Campbell et al. (2007) found maternal sensitivity to decline as depressive symptoms increased, whereas Cents et al. (2013), Giallo et al. (2015), Kingston et al. (2018), and Van Der Waerden et al. (2015) highlighted the association between maternal subclinical depressive symptoms and an increase in children's problem behaviours.

The effects of paternal depressive symptoms on the child's emotional development have received far less attention. In the ALSPAC cohort, paternal depression during the postnatal period was associated with disadvantageous emotional and behavioural symptoms in children aged 3.5 years (Ramchandani, Stein, Evans, \& O'Connor, 2005). Boys had conduct problems, in particular, even after adjusting for any maternal postnatal depression and later paternal depression. In most situations, however, the fathers' mental health problems seem to have a smaller influence on young children than do mental health problems of the mother; this is probably because the mother is usually the primary caregiver (meaning that the child may be affected more by disruptions in parenting caused by maternal depression) (Smith, 2004). Connell and Goodman (2002) conducted a meta-analysis on the associations between parental mental disorders and internalizing and externalizing symptoms across childhood in their offspring. They reported that the younger the children were, the smaller was the influence of paternal depression; maternal depression had a greater significance for the child's internalizing problem behaviours.

Maternal and paternal depression are often comorbid: fathers have a heightened risk of depression when the mother is depressed (Fisher, 2017; Paulson \& Bazemore, 2010). Paternal involvement may moderate the effects of maternal depression on children's emotional problems; depending on the level of paternal depressive symptoms, and on their time spent with the child, fathers can either ameliorate or cause deterioration in the child's adverse outcomes (Mezulis, Hyde, \& Clark, 2004). Interestingly, GutierrezGalve, Stein, Hanington, Heron, and Ramchandani (2015) report that two thirds of the association between paternal depression and child emotional problems was mediated by family atmosphere and maternal depression, whereas maternal depression seemed to be associated with child outcomes via direct mother-child interactions. More studies are needed, however, concerning paternal depressive symptom trajectories and the effects of simultaneous persistent perinatal depressive symptoms of both parents on children's emotional problems.

Here, we investigate associations of maternal, paternal and combined parental longitudinal depressive symptom trajectories from pregnancy to 2 years postnatally with children's externalizing and internalizing symptoms at those ages. Based on the literature, we hypothesize that maternal depressive symptoms have a stronger impact on children's emotional problems than do paternal depressive symptoms at both time-points and that the combined effect of having two depressive parents is particularly harmful at both ages.

\section{Methods \\ Participants}

This study is part of the Finnish population-based CHILDSLEEP birth cohort. For its survey design details, see Paavonen et al. (2017). The ethics committee board of Pirkanmaa Hospital District (PHD) approved the CHILD-SLEEP study protocol (R11032), and participants with a written informed consent were eligible for the study. Recruitment of mothers and fathers involved maternity clinics in the PHD catchment area; for this study, we used maternal and paternal questionnaires at gestational week 32 (1,667 women and 1,598 men covering $74.3 \%$ of the families approved to receive the prenatal questionnaire), and at the 3-month $(1,421$ women; 1,343 men), 8-month (1,299 women; 1,211 men) and 2-year (1,038 women; 776 men) time-points. Children's questionnaires were completed by either parent: 949 children participated at the 2-year time-point, and 714 (of whom 128 had not participated at the 2-year time-point) participated at the 5-year time-point. At 2 years, we excluded five twins and five children with serious medical illnesses or syndromes (Down's syndrome, developmental disability), leaving a final sample of 939 children. At 5 years, we excluded eight twins and six children with serious medical illnesses or syndromes, leaving a final sample size of 700 . Children's mean age at the time of the 2-year questionnaire was 2.04 years (range 1.88-2.80, SD 0.11 ) and at the 5-year questionnaire 5.71 years (range 4.83$6.83, S D$ 0.43). At 2 years, $645(68 \%)$ of BITSEA questionnaires were completed by mothers, $9(0.9 \%)$ by fathers, 286 $(30 \%)$ by parents together, and $0.9 \%$ (9) answers were missing. At 5 years, 490 (69.4\%) questionnaires were completed by mothers, $8(1.1 \%)$ by fathers and $174(24.6 \%)$ by both parents.

Background information including maternal and paternal ages, education, net income and number of previous children was on the prenatal questionnaires (Table 1). 
Table 1 Characteristics of the individuals taking part

\begin{tabular}{|c|c|c|}
\hline & $\begin{array}{c}\text { Completed } \\
\text { BITSEA } \\
\text { At 2-year } \\
\text { follow-up } \\
\text { Mean }(S D) / \\
N(\%)\end{array}$ & $\begin{array}{c}\text { Completed } \\
\text { SDQ/FTF } \\
\text { At 5-year } \\
\text { follow-up } \\
\text { Mean }(S D) / \\
N(\%)\end{array}$ \\
\hline Maternal characteristics & $N=937$ & $N=700$ \\
\hline Age (pre) & $31.09(4.36)$ & $31.18(4.59)$ \\
\hline \multicolumn{3}{|l|}{ Education level } \\
\hline $\begin{array}{l}\text { None or some vocational } \\
\text { training }\end{array}$ & $39(4.3 \%)$ & $31(4.5 \%)$ \\
\hline $\begin{array}{l}\text { Vocational or polytechnic } \\
\text { degree }\end{array}$ & $520(56.7 \%)$ & $400(58.7 \%)$ \\
\hline University & $358(39.0 \%)$ & $251(36.8 \%)$ \\
\hline \multicolumn{3}{|l|}{ Income $(\text { pre })^{\mathrm{d}}$} \\
\hline$<1,000 €$ & $192(20.8 \%)$ & $140(20.4 \%)$ \\
\hline $1,000-2,000 €$ & 470 (51.0\%) & 355 (51.7\%) \\
\hline$>2,000 €$ & $260(28.2 \%)$ & $192(27.9 \%)$ \\
\hline \multicolumn{3}{|c|}{ Number of previous children } \\
\hline 0 & $438(50.0 \%)$ & $328(51.0 \%)$ \\
\hline 1 & 296 (33.8\%) & 208 (32.3\%) \\
\hline$\geq 2$ & $142(16.2 \%)$ & 107 (16.6\%) \\
\hline Paternal characteristics & $N=895$ & $N=671$ \\
\hline Age (pre) (years) & $32.89(4.85)$ & $32.78(5.1)$ \\
\hline \multicolumn{3}{|l|}{ Education level } \\
\hline $\begin{array}{l}\text { None or some vocational } \\
\text { training }\end{array}$ & $83(9.5 \%)$ & $62(9.4 \%)$ \\
\hline $\begin{array}{l}\text { Vocational or polytechnic } \\
\text { degree }\end{array}$ & 493 (56.5\%) & $379(57.7 \%)$ \\
\hline University & $297(34.0 \%)$ & 216 (32.9\%) \\
\hline \multicolumn{3}{|l|}{ Income (pre) ${ }^{\mathrm{d}}$} \\
\hline$<1,000 €$ & $54(6.1 \%)$ & $45(6.8 \%)$ \\
\hline $1,000-2,000 €$ & $279(31.6 \%)$ & 206 (31.2\%) \\
\hline$>2,000 €$ & $550(62.3 \%)$ & $410(62.0 \%)$ \\
\hline Child's characteristics & $N=939$ & $N=700$ \\
\hline \multicolumn{3}{|l|}{ Gender } \\
\hline Girl & $442(47.1 \%)$ & $332(47.4 \%)$ \\
\hline Boy & 497 (52.9\%) & $368(52.6 \%)$ \\
\hline $\begin{array}{l}\text { Gestational age at } \\
\text { birth (weeks) }\end{array}$ & $40.01(1.27)$ & $40.01(1.21)$ \\
\hline Birth weight (g) & $3,575(451)$ & $3,582(450)$ \\
\hline BITSEA total problems & $9.17(5.24)$ & \\
\hline $\begin{array}{l}\text { BITSEA externalizing } \\
\text { problems }\end{array}$ & $3.08(2.17)$ & \\
\hline $\begin{array}{l}\text { BITSEA internalizing } \\
\text { problems }\end{array}$ & $3.23(2.85)$ & \\
\hline SDQ total & & $8.03(4.40)$ \\
\hline SDQ conduct problems & & $1.96(1.58)$ \\
\hline SDQ emotional problems & & $1.38(1.48)$ \\
\hline FTF total & & $4.32(4.38)$ \\
\hline FTF externalizing problems & & $2.81(3.22)$ \\
\hline FTF internalizing problems & & $1.51(1.79)$ \\
\hline
\end{tabular}

Pre refers to prenatal questionnaire at gestational week 32 . BITSEA, The Brief Infant-Toddler Social and Emotional Assessment; SDQ, The Strengths and Difficulties Questionnaire; FTF, the Five to Fifteen questionnaire.

${ }^{\mathrm{a}}$ Income after taxes per month.

\section{Measures}

Parental depressive symptoms. Depressive symptoms were measured as part of the maternal and paternal questionnaires prenatally and again completed at the child's age of 3 and 8 months, and 2 years. It incorporated the 10item version of the Center for Epidemiological Studies Depression Scale, CES-D (Irwin, Artin, \& Oxman, 1999; Radloff, 1977). Ratings for each item were on a four-point Likert-type scale, and two of the questions were reverse-scored. When each total score was calculated, a higher score indicated greater depressive symptoms (range 0-30).

Children's emotional problems at the age of 2 years. We used children's social- and emotional-problem questions (31 items, scale range 0-62) from the Brief Infant-Toddler Social and Emotional Assessment (BITSEA) (Briggs-Gowan, Carter, Irwin, Wachtel, \& Cicchetti, 2004). Each item was rated on a three-point Likert scale. The problem domain included externalizing (seven items) and internalizing (14 items) subdomains. Total problem, externalizing problem and internalizing problem scores were calculated.

Children's emotional symptoms at the age of 5 years. We used the total difficulties score $(20$ items, scale range 0-40) of the Strengths and Difficulties Questionnaire (SDQ) screening tool (Goodman, 1997), which comprises the subscales of emotional- (five items), conduct- (five items), hyperactivity/inattention- (five items) and peer-relationship problems (five items). Furthermore, we also report the emotional (internalizing)- and conduct-problem subscales separately. Parents rated the statements on a three-point scale with five of the items reverse-scored.

In addition, we used the risk of emotional/behavioural problems subscale (25 items; FTF total, scale range $0-50$ ) of the Five to Fifteen (FTF) (Kadesjö et al., 2004) parent-reported questionnaire. This subscale can be further divided into risk of internalizing problems (12 items) and externalizing problems (13 items), with three response alternatives for each item. The FTF is a long questionnaire with 181 items and three response options for each variable. It comprises items regarding development and psychosocial symptoms. It has eight subscales, such as motor and verbal skills, executive functioning, attention, memory, social skills, and emotional and behavioural symptoms. The items in the symptom scales are based on the DSM-IV items.

\section{Statistical analyses}

The parents were grouped by means of a latent profile analysis according to their longitudinal patterns of depressive symptoms. A three-class solution with relatively stable depressive symptom profiles (stable low, stable intermediate and stable high depressive symptoms) for both mothers and fathers was considered most informative, clinically sound, and with an acceptable fit to the data. Detailed parental depressive symptom trajectories are available elsewhere (Kiviruusu et al., 2019). We constructed four combined parental depressive symptom groups based on these parental longitudinal depressive symptom trajectories (Table 2). In Group 1, maternal (hereafter mat) and paternal (hereafter pat) depressive symptoms were low (Mat-, Pat-). In Group 2, maternal depressive symptoms were persistently intermediate/high and paternal symptoms low (Mat+, Pat-). In Group 3, paternal depressive symptoms were persistently intermediate/high and maternal symptoms were low (Mat-, Pat + ). And in Group 4, both maternal and paternal depressive symptoms were persistently intermediate/high (Mat+, Pat + ).

Computed general linear models utilized children's emotional-symptom scores (BITSEA total/externalizing/internalizing problems, SDQ total/conduct/internalizing problems and FTF total/externalizing/internalizing problems) as the dependent variable and combined parental depressive symptom groups as the grouping variable. Almost all outcome variables were somewhat skewed to the right, and because of this, we did sensitivity analyses with nonparametric testing and after normality transformations of dependent variables. The findings were virtually unchanged. We therefore present the original results. 
Table 2 Combined maternal and paternal trajectory group CES-D scores at 2 and 5 years

\begin{tabular}{|c|c|c|c|c|}
\hline & Trajectory group & $N(\%)$ & Mat CES-D & Pat CES-D \\
\hline \multirow[t]{4}{*}{ At 2 years, $N=902$} & Group 1 (Mat-, Pat-) & $490(54.3)$ & $\leq 3.62$ & $\leq 3.34$ \\
\hline & Group 2 (Mat+, Pat-) & 187 (20.7) & $7.25-8.73$ & $3.01-3.40$ \\
\hline & Group 3 (Mat-, Pat+) & $102(11.3)$ & 2.99-4.17 & $6.68-8.40$ \\
\hline & Group 4 (Mat+, Pat+) & $123(13.6)$ & $\geq 8.19$ & $\geq 7.51$ \\
\hline \multirow[t]{3}{*}{ At 5 years, $N=675$} & Group 1 (Mat-, Pat-) & $353(52.3)$ & $\leq 3.66$ & $\leq 3.28$ \\
\hline & Group 2 (Mat+, Pat-) & $134(19.8)$ & 7.07-8.96 & $2.98-3.50$ \\
\hline & Group 4 (Mat+, Pat+) & 99 (14.7) & $\geq 8.04$ & $\geq 7.55$ \\
\hline
\end{tabular}

Mat, maternal; Pat, paternal; CES-D, The Center for Epidemiological Studies Depression Scale.

Separate analyses were performed at 2-year and 5-year time-points. In the post hoc comparisons of these unadjusted models, we used the Bonferroni or the Tamhane test depending on the homogeneity of the variances in the parental groups. Interaction terms between child gender and parental depressive symptom groups were investigated separately to study gender-dependent effects of parental depression.

Next, we computed adjusted general linear models. The combined parental depressive symptom groups served as the main explanatory variables, while children's emotional problems at the 2-and 5-year time-points were adjusted for maternal age, maternal education, paternal age, paternal education, maternal income, paternal income and number of previous children (maternal). Second, analyses were also adjusted for child's gestational age, birthweight, gender and age at the time when the questionnaire was answered (BITSEA or FTF questionnaire). The Bonferroni test served for post hoc comparisons.

Finally, a general linear model served to analyse separate maternal and paternal three-class depressive symptom trajectories by use of the same child emotional-problem outcomes as the dependent variable and child gender as the sole covariate. IBM SPSS Statistics 25 (IBM Corp, Armonk, NY, USA) was used in all analyses.

The missing answers in the subscales were imputed by the individual mean if at least $70 \%$ of the items were available. Otherwise, the subscale score was considered missing. Such missingness was very infrequent in this data set (approximately $2 \%$ of answers per item were missing at age two and $5 \%$ at age five).

\section{Results}

For descriptive statistics of the sample and of BITSEA, SDQ and FTF questionnaires, see Table 1.

\section{Combined maternal and paternal depressive symptom trajectories and children's emotional problems at the age of 2 years}

As is clear in Figure 1, statistically significant differences in BITSEA scores appeared for different parental depressive symptom groups (also see Table 3). In the adjusted models, effect sizes were medium for total (partial eta squared 0.105) and internalizing problems (0.081) and small for externalizing problems (0.035). Post hoc comparisons indicated that children whose mothers reported elevated depressive symptoms (Group 2) and children with two depressive parents (Group 4) received significantly higher scores on all three BITSEA scales than children with two nondepressed parents (Group 1). Mean scores for Group 2 did not differ significantly from scores for Group 4 in any of the analyses. Importantly, children in Group 3, that is with only a father reporting depressive symptoms, did not differ significantly in BITSEA scores from children in Group 1.

\section{Combined maternal and paternal depressive symptom trajectories and children's emotional problems at age five}

As Figure 2 illustrates, statistically significant differences in SDQ and FTF scores existed for different parental depressive symptom groups (also see Table 3). In the adjusted models, effect sizes for the SDQ total problem (partial eta squared 0.079) and FTF internalizing (0.060) problems were medium, whereas all the other effect sizes were small. Post hoc comparisons in the adjusted models indicated that mean scores for the group with a depressive mother (Group 2) and two depressive parents (Group 4) were significantly higher than scores for the group with two nondepressive parents (Group 1) concerning children's total problems, conduct and internalizing problems measured by the SDQ questionnaire, and total and internalizing problems measured by the FTF questionnaire. Mean scores for FTF externalizing problems were significantly higher for Group 4 than for Group 1 (Table 3).

\section{Separate three-class maternal and paternal depressive symptom trajectories and children's emotional problems at ages two and five}

In Figures S1-S3, and in Table S1, we demonstrate how maternal and paternal depressive symptom trajectories separately associate with their children's emotional problems at two and five. A higher level of maternal depressive symptoms was associated with a higher level of children's emotional problems at both ages, except for internalizing symptoms at age five. Effect sizes were approaching the category large in regard to maternal depressive symptoms and children's total (partial eta squared 

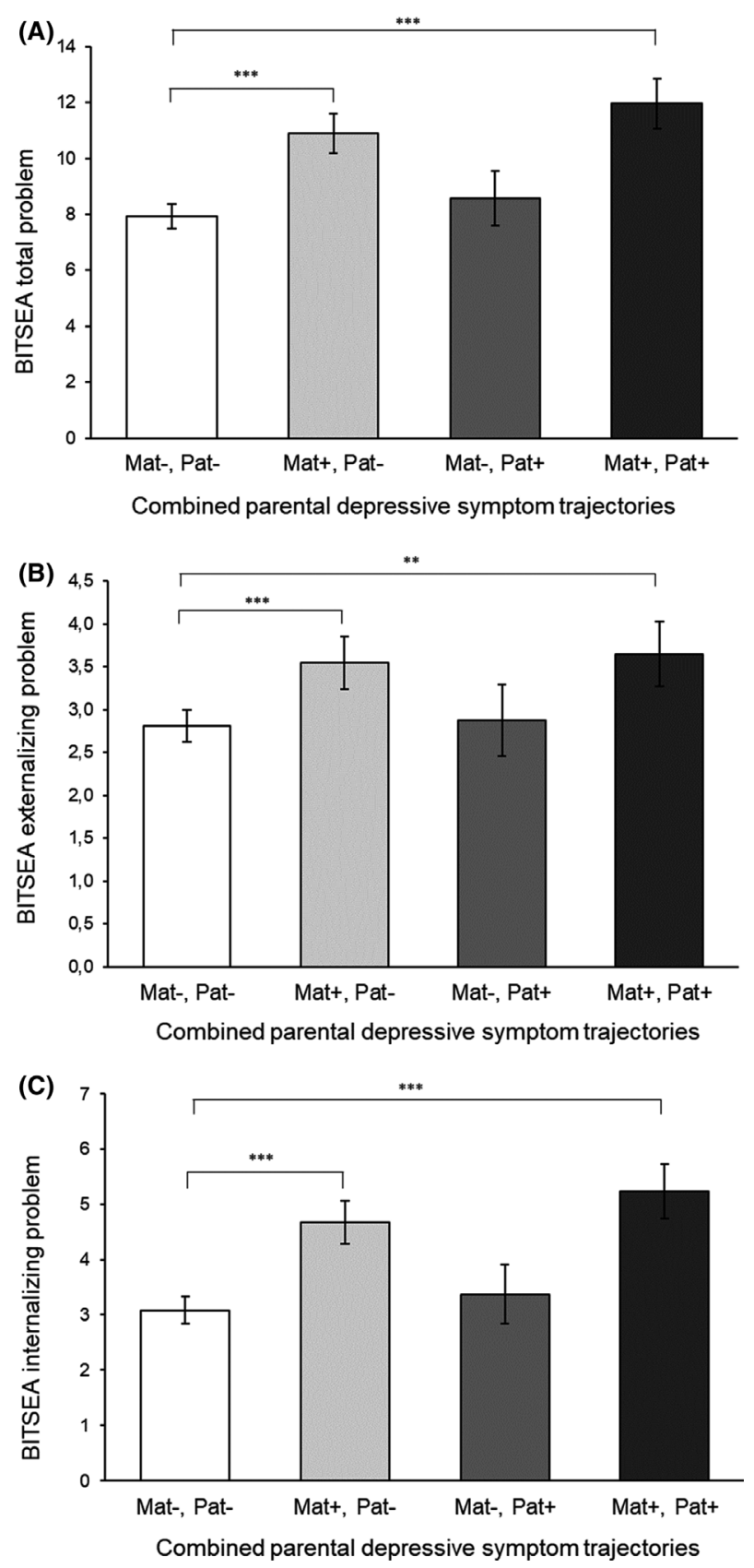

Figure 1 Combined parental depressive symptom trajectories versus children's $(A)$ total, $(B)$ externalizing and $(C)$ internalizing emotional problems at the age of 2 years measured by Brief Infant-Toddler Social and Emotional Assessment (BITSEA). Note. Error bars indicate 95\% confidence intervals, and asterisks indicate difference between the mean scores when compared to nondepressive (Mat-, Pat-) group. ${ }^{* * *} p<.001,{ }^{* *} p<.01$, $* p<.05$; Mat, maternal; Pat, paternal

$0.101)$ and internalizing problems $(0.095)$ at 2 years, whereas other effect sizes were small.

As paternal depressive symptoms increased, there occurred no similar trend towards an increase in children's emotional problems, in contrast to that seen in mothers. Effect sizes for paternal trajectories were considerably smaller, and externalizing problems at the age of two as well as internalizing problems at age five were not associated with paternal depressive symptom trajectories.
Interaction effects between child gender and all parental depressive symptom trajectory groups were nonsignificant in all analyses.

\section{Discussion}

To our knowledge, this is the first or one of the first studies combining both parental depressive symptom trajectories in risk analysis. We found that maternal persistent depressive symptoms were associated with children's externalizing and internalizing emotional problems at both 2 and 5 years, but if the mother was nondepressive, paternal depressive symptoms were not associated with children's outcomes in any of the adjusted post hoc analyses. This finding was similar both when the parental depressive symptom trajectories were combined and when they were analysed separately. Contrary to our hypothesis, having two depressive parents did not correlate with an increased risk for these children's emotional problems when compared to risk for those offspring having only a mother with depressive symptoms.

These children's emotional problems increased gradually along with maternal depression severity, with the exception of internalizing problems at the age of five. Concerning a father's higher level of depressive symptoms, however, no such an association emerged.

Whether parental depressive symptoms differently influence boys and girls, findings have been mixed (Goodman et al., 2011). Our findings lacked any statistically significant interactions between parental depressive symptom trajectories and children's gender.

Our findings were very similar to those of Generation R cohort (Cents et al., 2013; Meaney, 2018), because in both studies, higher levels of maternal depressive symptoms were related to respective increases in children's externalizing and internalizing problems. In addition, we also assessed paternal and combined parental longitudinal depressive symptom groups and their associations with child outcomes, an area in which earlier studies are sparse, and importantly, were able to confirm the significance of maternal depression independent of father's mental well-being.

The mechanisms by which parental depressive symptoms affect child outcomes have long been under discussion. Whereas genetic vulnerability (Hannigan et al., 2018) plays a role, interaction between child and parent is considered to be the primary modifiable mechanism mediating the effects of parental depression to the offspring (Stein et al., 2014). The connection is, however, reciprocal, so that maternal perinatal depressive symptoms and child emotional difficulties, especially externalizing symptoms that overtly challenge parent-child interaction, can negatively reinforce each other (Meaney, 2018). 


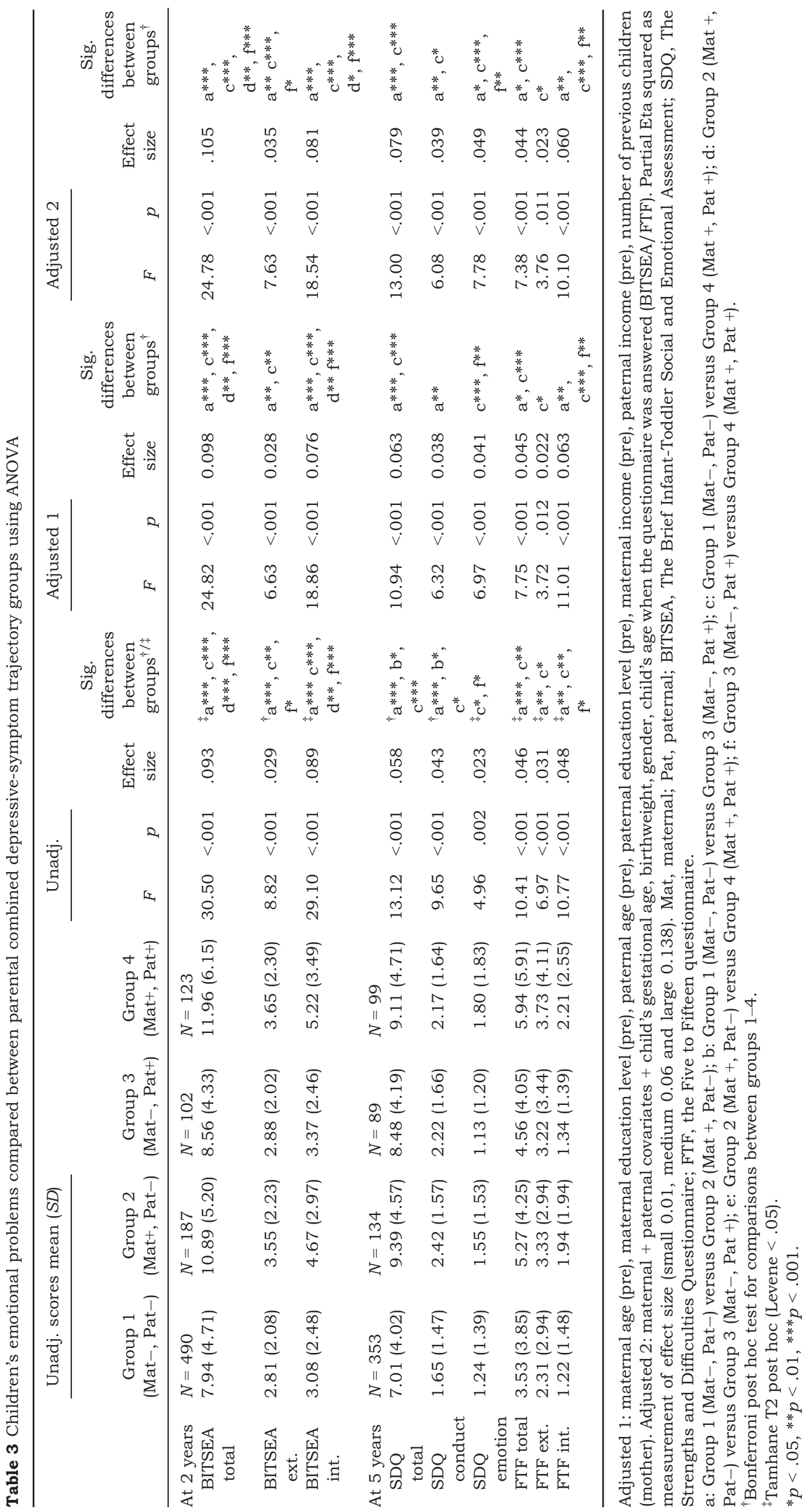




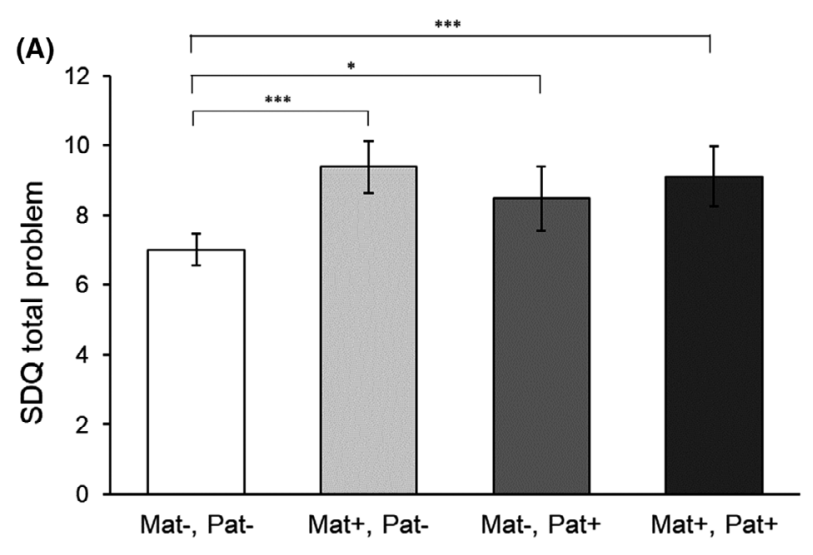

Combined parental depressive symptom trajectories
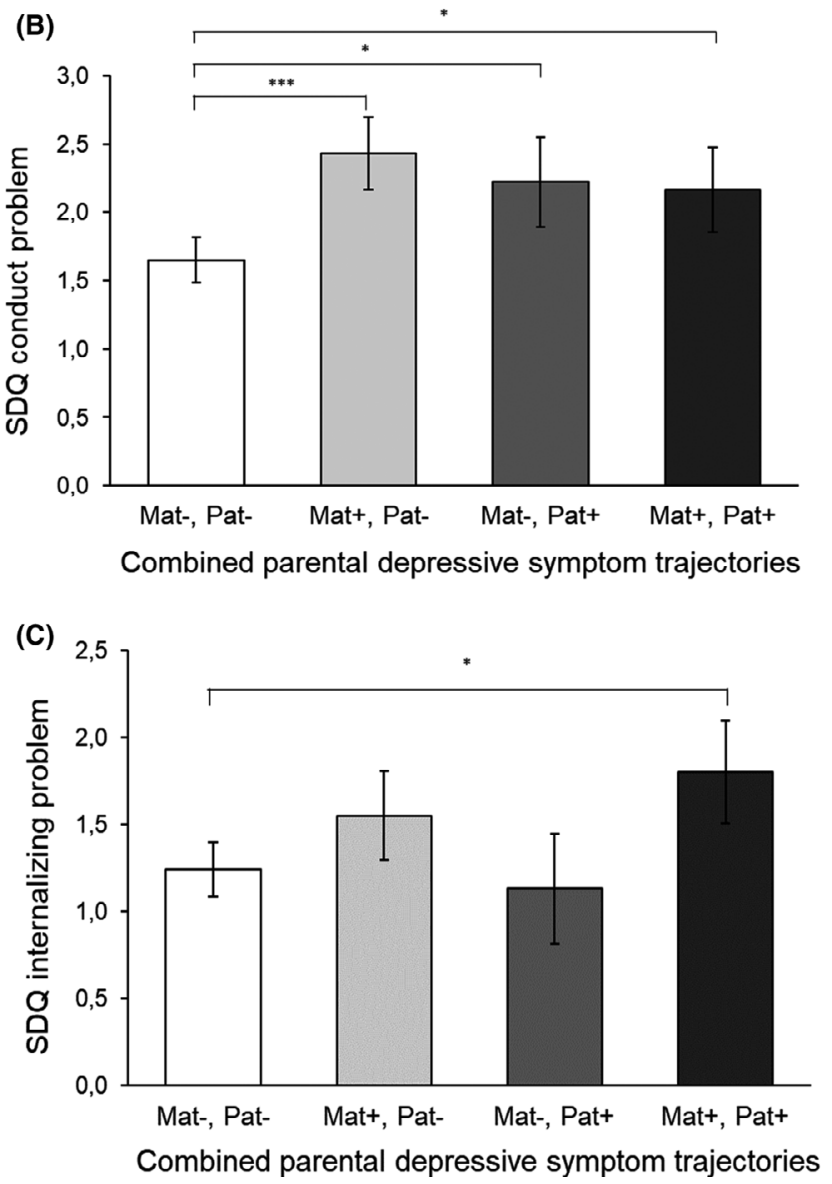

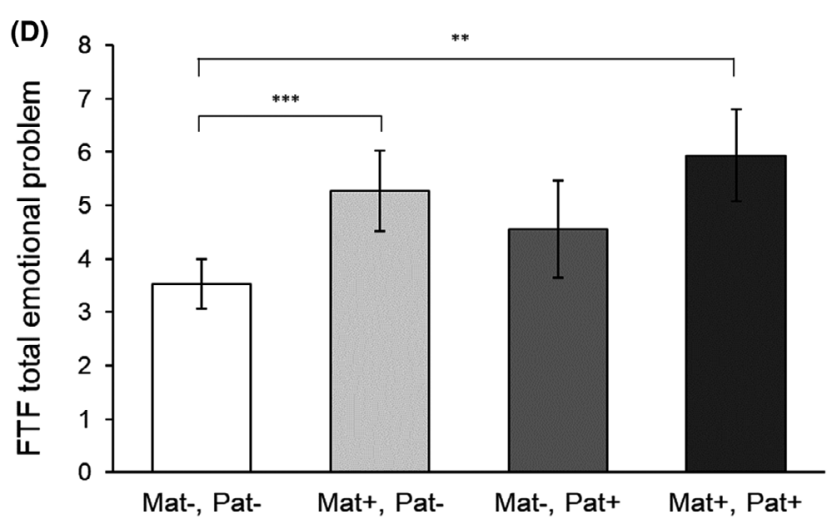

Combined parental depressive symptom trajectories

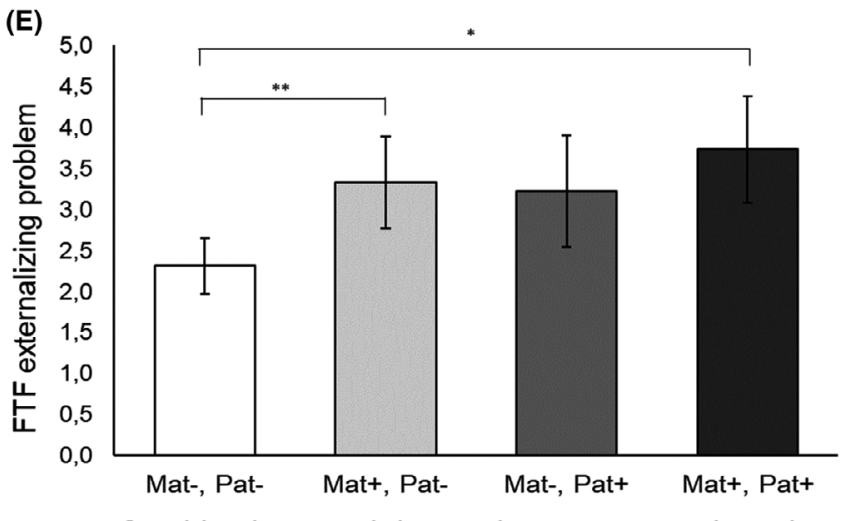

Combined parental depressive symptom trajectories

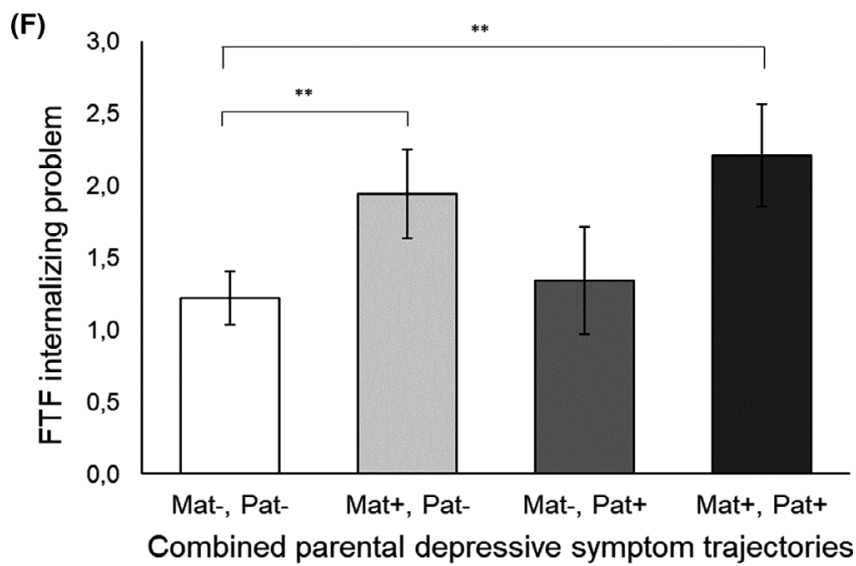

Figure 2 Combined parental depressive symptom trajectory groups versus children's emotional problems at the age of 5 years measured by Strengths and Difficulties (SDQ) and Five to Fifteen (FTF) questionnaires. (A) SDQ total, (B) SDQ conduct, (C) SDQ internalizing, (D) FTF total emotional, (E) FTF externalizing and (F) FTF internalizing emotional problems. Note. Error bars indicate $95 \% \mathrm{Cl}$; asterisks indicate difference between the mean scores when compared to 'Mat-, Pat-' group. ${ }^{* * *} p<.001,{ }^{* *} p<.01,{ }^{*} p<.05$; Mat, maternal; Pat, paternal

Subclinical levels of depressive symptoms (e.g. $7<$ CES-D < 10) have raised interest lately. We found an intermediate level of maternal depressive symptoms to be associated with an intermediate level of children's emotional problems in separate three-class maternal depressive symptom trajectories. Interestingly, Meaney pointed out in his recent review (Meaney, 2018) the importance of heightened subclinical levels of maternal depressive symptoms: impairments in parenting and psychosocial functioning are as severe as in women with clinical depression (Weinberg et al., 2001).

Certain features of the scales should be noted when interpreting our results. First, the two scales at the 5year time-point assess slightly different aspects of emotional problems. For example, the conduct scale of SDQ measures only conduct symptoms (five items), whereas the externalizing scale of FTF (13 items) follows relatively closely criteria for DSM-IV oppositional defiant disorder. Second, concerning measurement of parental depression, Brownhill, Wilhelm, 
Barclay, and Schmied (2005) have argued that traditional depression scales may underestimate paternal depressive symptoms, because fathers may have more externalizing symptoms as well as substance abuse as signs of psychiatric ill-being instead of depressive symptoms than would mothers.

Goodman and Gotlib (1999) have proposed that healthy, involved and supportive fathers may have a protective effect on children of depressed mothers. Having an absent father or a second parent with psychopathology would, however, have an accumulating adverse effect, as the child loses the potentially 'protective' healthy parent. In our population-based sample, we observed no accumulative effect of parental depressive symptoms; what remains to be clarified is whether such influences will become evident during later stages of offspring development. Indeed, Connell and Goodman (2002) found in their meta-analysis paternal depression and alcoholism to have a greater effect in series comprising older children.

Our study has a few limitations. We assessed parental depressive symptom status and child outcomes using parental questionnaires instead of structured clinical interviews, and we lacked an informant outside the family. In a meta-analysis by Goodman et al. (2011), a clinical diagnosis of maternal depression showed, however, similar effect sizes concerning child outcomes as those of self-assessed depression questionnaires. The Goodman group (2011) also state that indeed, concerning children's emotional problems, studies relying on mothers' reports found somewhat larger effect sizes when compared to findings in reports by teachers, others, maternal-child dyads or child self-reports. Brennan et al. (2000) argue, however, that maternal observations should not be overlooked; and as Richters (1992) suggests, several studies have shown that depressed women are more accurate in detecting true disorders in their children than are nondepressed women. Noticing a child's internalizing emotional problems may also be difficult - for instance, in the day care environment.

As a limitation, some of the families dropped out of the study. We compared the parents of the children who did not respond at the 2-year time-point to the parents of other children (those who had responses both at 2 and 5 years and at 2 years). In ANOVA, however, this was unrelated to parental prenatal depression (both $p$-values $>0.3$ ). As another limitation, fathers' attrition rate was higher than that of mothers. However, in the latent profile analyses (Kiviruusu et al., 2019) the profiles were analysed with full information maximum likelihood estimation, which is currently the best available method (in addition to multiple imputation) to deal with missing information due to attrition. Thus, the profiles were available for all fathers and mothers who had at least one CES-D score (out of four possible measurement points), which means that they were available for practically all of them. The fathers' attrition thus did not affect the analyses of this paper. Furthermore, inclusion of 5-year parental depressive symptom figures could have made more evident the association between parental concurrent symptoms and child outcome. However, our aim was to study whether early parental depression influenced the development of children. Concerning three-class paternal trajectories, the number of fathers in the highest trajectory groups was small when compared to number of mothers.

The strengths of the study are its representative and relatively large sample and its longitudinal study design which allowed us to construct trajectories of both maternal and paternal depressive symptoms and to compare the contribution of longitudinal parental depressive symptoms with children's outcomes. Assessment of children both at 2 and 5 years and use of two different questionnaires at 5 years can also count as a strength.

\section{Conclusion}

To conclude, maternal depressive symptoms were associated with children's total, externalizing and internalizing problems to a gradually increasing degree, whereas paternal depressive symptoms did not show a similar pattern. It seems that absence of depressive symptoms in the fathers did not protect the child from the adverse effect of maternal depressive symptoms. These findings also should lead us to place more emphasis on the role of maternal subclinical depressive symptoms as a risk for the emotional development of the child. To prevent the intergenerational transmission of emotional problems, we recommend early detection, intervention and effective treatment of maternal depressive symptoms preferably already during pregnancy. From an economic perspective, the costs of prenatal interventions for families at risk would probably be lower and more easily applied than cost of parent-child interventions targeting children after the child's emotional disturbance becomes apparent.

\section{Supporting information}

Additional supporting information may be found online in the Supporting Information section at the end of the article:

Figure S1. Maternal and Paternal depressive symptom trajectories versus children's socioemotional problems at the age of 2 years.

Figure S2. Maternal and Paternal depressive symptom trajectories versus children's emotional problems at 5 years measured by Strengths and Difficulties (SDQ).

Figure S3. Maternal and Paternal depressive symptom trajectories versus children's emotional problems at the age of 5 years measured by Five to Fifteen (FTF).

Table s1. Children's emotional problems compared between separate maternal and paternal three-class depressive symptom trajectory groups using general linear model. 


\section{Acknowledgements}

This work was supported by the Academy of Finland (\# 134880 and 253346 TP; \# 308588 EJP, \# 277557 O.S.H. \# 317080 A.K.), the Foundation for Pediatric Research (E.J.P., J.T.P.), Competitive Research Financing of the Expert Responsibility Area of Helsinki University Hospital (J.T.P.), the Emil Aaltonen Foundation (J.T.P.), the Finnish Psychiatric Association (J.T.P.), the Jalmari and Rauha Ahokas Foundation (J.T.P.), and the Gyllenberg Foundation (T.P.), Yrjö Jahnson Foundation (E.J.P.), Finnish Cultural Foundation (E.J.P.), Competitive Research Financing of the Expert Responsibility Area of Tampere University Hospital (O.S.H.), the Arvo and Lea Ylppö Foundation (O.S.H.) and the Doctors' Association in Tampere (O.S.H.). The authors have declared that they have no competing or potential conflicts of interest.

\section{Author contributions}

E.J.P., T.P., A.K., P.P. and O.S.H. designed the study. J.T.P., O.K. and E.J.P. were primarily responsible for data analysis and writing of the article. A.K., O.S.H., T.P. and P.P. contributed critically to the writing of the article.

\section{Correspondence}

Johanna T. Pietikäinen, Department of Public Health Solutions, National Institue for Health and Welfare, Mannerheimintie 168, P .O. Box 30, 00271 Helsinki, Finland; Email: johanna.t.pietikainen@helsinki.fi

\section{Key points}

- Parental depressive symptoms are a risk factor for children's emotional problems, but only a few studies have addressed how simultaneous and persistent maternal and paternal depressive symptoms contribute to children's emotional outcomes.

- We investigated how parental depressive symptom trajectories (from late pregnancy until 2 years after delivery) were associated with children's emotional symptoms at the age of two $(N=939)$ and five $(N=700)$ years.

- Paternal depressive symptoms were associated with children's emotional problems only if the mother was also depressive.

- Nondepressive fathers did not protect children from adverse effects of maternal depressive symptoms.

- Intermediate levels of maternal depressive symptoms were associated with children's emotional problems at both the 2- and 5-year time-points.

\section{References}

Bagner, D.M., Pettit, J.W., Lewinsohn, P.M., \& Seeley, J.R. (2010). Effect of maternal depression on child behavior: A sensitive period? Journal of the American Academy of Child and Adolescent Psychiatry, 49, 699-707.

Brennan, P.A., Hammen, C., Andersen, M.J., Bor, W., Najman, J.M., \& Williams, G.M. (2000). Chronicity, severity, and timing of maternal depressive symptoms: Relationships with child outcomes at age 5. Developmental Psychology, 36, 759-766.

Briggs-Gowan, M.J., Carter, A.S., Irwin, J.R., Wachtel, K., \& Cicchetti, D.V. (2004). The brief infant-toddler social and emotional assessment: Screening for social-emotional problems and delays in competence. Journal of Pediatric Psychology, 28, 143-155.

Brownhill, S., Wilhelm, K., Barclay, L., \& Schmied, V. (2005). "Big build": Hidden depression in men. Australian and New Zealand Journal of Psychiatry, 39, 921-931.

Cameron, E.E., Sedov, I.D., \& Tomfohr-Madsen, L.M. (2016). Prevalence of paternal depression in pregnancy and the postpartum: An updated meta-analysis. Journal of Affective Disorders, 206, 189-203.

Campbell, S.B., Matestic, P., von Stauffenberg, C., Mohan, R., \& Kirchner, T. (2007). Trajectories of maternal depressive symptoms, maternal sensitivity, and children's functioning at school entry. Developmental Psychology, 43, 1202-1215.

Campbell, S.B., Morgan-Lopez, A.A., Cox, M.J., \& McLoyd, V.C. (2009). A latent class analysis of maternal depressive symptoms over 12 years and offspring adjustment in adolescence. Journal of Abnormal Psychology, 118, 479-493.

Cents, R.A.M., Diamantopoulou, S., Hudziak, J.J., Jaddoe, V.W.V., Hofman, A., Verhulst, F.C., . . \& Tiemeier, H. (2013). Trajectories of maternal depressive symptoms predict child problem behaviour: The Generation R Study. Psychological Medicine, 43, 13-25.

Connell, A.M., \& Goodman, S.H. (2002). The association between psychopathology in fathers versus mothers and children's internalizing and externalizing behavior problems: A meta-analysis. Psychological Bulletin, 18, 746-773.

England, M.J., \& Sim, L.J. (2009). Depression in parents, parenting, and children: Opportunities to improve identification, treatment, and prevention (pp. 17-42). Washington, DC: National Academies Press.

Fisher, S.D. (2017). Paternal mental health: Why is it relevant? American Journal of Lifestyle Medicine, 11, 200-211.

Giallo, R., Woolhouse, H., Gartland, D., Hiscock, H., \& Brown, S. (2015). The emotional-behavioural functioning of children exposed to maternal depressive symptoms across pregnancy and early childhood: A prospective Australian pregnancy cohort study. European Child and Adolescent Psychiatry, 24, 1233-1244.

Goodman, R. (1997). The strengths and difficulties questionnaire: A research note. Journal of Child Psychology and Psychiatry, 38, 581-586.

Goodman, S.H., \& Gotlib, I.H. (1999). Risk for psychopathology in the children of depressed mothers: A developmental 
model for understanding mechanisms of transmission. Psychological Review, 106, 458-490.

Goodman, S.H., Rouse, M.H., Connell, A.M., Broth, M.R., Hall, C.M., \& Heyward, D. (2011). Maternal depression and child psychopathology: A meta-analytic review. Clinical Child and Family Psychology Review, 14, 1-27.

Gutierrez-Galve, L., Stein, A., Hanington, L., Heron, J., \& Ramchandani, P. (2015). Paternal depression in the postnatal period and child development: Mediators and moderators. Pediatrics, 135, e339-e347.

Hannigan, L.J., Eilertsen, E.M., Gjerde, L.C., Reichborn-Kjennerud, T., Eley, T.C., Rijsdijk, F.V., ... \& McAdams, T.A. (2018). Maternal prenatal depressive symptoms and risk for early-life psychopathology in offspring: Genetic analyses in the Norwegian Mother and Child Birth Cohort Study. The Lancet Psychiatry, 5, 808-815.

Irwin, M., Artin, K.H., \& Oxman, M.N. (1999). Screening for depression in the older adult: Criterion validity of the 10item Center for Epidemiological Studies Depression Scale (CES-D). Archives of Internal Medicine, 159, 1701-1704.

Kadesjö, B., Janols, L.O., Korkman, M., Mickelsson, K., Strand, G., Trillingsgaard, A., \& Gillberg, C. (2004). The FTF (Five to Fifteen): The development of a parent questionnaire for the assessment of ADHD and comorbid conditions. European Child and Adolescent Psychiatry, Supplement, 3, $13 \mathrm{III} / 3-\mathrm{III} / 13$.

Kingston, D., Kehler, H., Austin, M.P., Mughal, M.K., Wajid, A., Vermeyden, L., . . \& Giallo, R. (2018). Trajectories of maternal depressive symptoms during pregnancy and the first 12 months postpartum and child externalizing and internalizing behavior at three years. PLoS ONE, 13, e0195365.

Kiviruusu, O., Pietikäinen, J.T., Kylliäinen, A., Pölkki, P., Saarenpää-Heikkilä, O., Marttunen, M., ... \& Paavonen, E.J. (2019). Trajectories of mothers' and fathers' depressive symptoms from pregnancy to 24 months postpartum. Journal of Affective Disorders, In press. https://doi.org/10. 1016/j.jad.2019.09.038.

Meaney, M.J. (2018). Perinatal maternal depressive symptoms as an issue for population health. American Journal of Psychiatry, 175, 1084-1093.

Mezulis, A.H., Hyde, J.S., \& Clark, R. (2004). Father involvement moderates the effect of maternal depression during a child's infancy on child behavior problems in kindergarten. Journal of Family Psychology, 18, 575-588.

Murray, L., Sinclair, D., Cooper, P., Ducournau, P., Turner, P., \& Stein, A. (1999). The socioemotional development of 5year-old children of postnatally depressed mothers. Journal of Child Psychology and Psychiatry, 40, 1259-1271.

O’Donnell, K.J., \& Meaney, M.J. (2017). Fetal origins of mental health: The developmental origins of health and disease hypothesis. American Journal of Psychiatry, 174, 319-328.

O’Hara, M.W., \& McCabe, J.E. (2013). Postpartum depression: Current status and future directions. Annual Review of Clinical Psychology, 9, 379-407.

Paavonen, E.J., Saarenpää-Heikkilä, O., Pölkki, P., Kylliäinen, A., Porkka-Heiskanen, T., \& Paunio, T. (2017). Maternal and paternal sleep during pregnancy in the Child-sleep birth cohort. Sleep Medicine, 29, 47-56.

Park, M., Brain, U., Grunau, R.E., Diamond, A., \& Oberlander, T.F. (2018). Maternal depression trajectories from pregnancy to 3 years postpartum are associated with children's behavior and executive functions at 3 and 6 years. Archives of Women's Mental Health, 21, 353-363.

Paulson, J.F., \& Bazemore, S.D. (2010). Prenatal and postpartum depression in fathers and its association with maternal depression: A meta-analysis. JAMA - Journal of the American Medical Association, 303, 1961-1969.

Radloff, L.S. (1977). The CES-D Scale: A Self-Report Depression Scale for research in the general population. Applied Psychological Measurement, 1, 385-401.

Ramchandani, P., Stein, A., Evans, J., \& O'Connor, T.G. (2005). Paternal depression in the postnatal period and child development: A prospective population study. The Lancet, 365, 2201-2205.

Richters, J.E. (1992). Depressed mothers as informants about their children: A critical review of the evidence for distortion. Psychological Bulletin, 112, 485-499.

Smith, M. (2004). Parental mental health: Disruptions to parenting and outcomes for children. Child and Family Social Work, 9, 3-11.

Stein, A., Pearson, R.M., Goodman, S.H., Rapa, E., Rahman, A., McCallum, M., ... \& Pariante, C.M. (2014). Effects of perinatal mental disorders on the fetus and child. Lancet, 384, 1800-1819.

Van Der Waerden, J., Galéra, C., Larroque, B., Saurel-Cubizolles, M.J., Sutter-Dallay, A.L., \& Melchior, M. (2015). Maternal depression trajectories and children's behavior at age 5 years. Journal of Pediatrics, 166, 1440-1448.

Weinberg, M.K., Tronick, E.Z., Beeghly, M., Olson, K.L., Kernan, H., \& Riley, J.M. (2001). Subsyndromal depressive symptoms and major depression in postpartum women. American Journal of Orthopsychiatry, 71, 87-97.

Weissman, M.M., Berry, O.O., Warner, V., Gameroff, M.J., Skipper, J., Talati, A., . . \& Wickramaratne, P. (2016). A 30year study of 3 generations at high risk and low risk for depression. JAMA Psychiatry, 73, 970-977.

Weissman, M.M., Pilowsky, D.J., Wickramaratne, P.J., Talati, A., Wisniewski, S.R., Fava, M., ... \& Rush, A.J. (2006). Remissions in maternal depression and child psychopathology: A STAR*D-child report. Journal of the American Medical Association, 295, 1389-1398.

Weissman, M.M., Wickramaratne, P., Gameroff, M.J., Warner, V., Pilowsky, D., Kohad, R.G., .. \& \& Talati, A. (2016). Offspring of depressed parents: 30 years later. American Journal of Psychiatry, 173, 1024-1032.

Weissman, M.M., Wickramaratne, P., Nomura, Y., Warner, V., Pilowsky, D., \& Verdeli, H. (2006). Offspring of depressed parents: 20 Years later. American Journal of Psychiatry, 163, 1001-1008.

Accepted for publication: 15 August 2019

First published online: 18 September 2019 